OPEN ACCESS

Edited by:

Feng Liu,

Tianjin Medical University General Hospital, China

Reviewed by:

Zhao Qing,

Nanjing Drum Tower Hospital, China

Yang Yang,

Fourth Military Medical University,

China

*Correspondence:

Lishan Qiao

qlishan@163.com

Specialty section:

This article was submitted to Brain Imaging Methods, a section of the journal Frontiers in Neuroscience

Received: 17 April 2021

Accepted: 26 July 2021

Published: 23 August 2021

Citation:

Guo T, Zhang Y, Xue Y, Qiao $L$ and Shen D (2021) Brain Function Network: Higher Order vs. More

Discrimination

Front. Neurosci. 15:696639, doi: 10.3389/fnins.2021.696639

\section{Brain Function Network: Higher Order vs. More Discrimination}

\author{
Tingting Guo ${ }^{1}$, Yining Zhang ${ }^{1}$, Yanfang Xue ${ }^{1}$, Lishan Qiao ${ }^{* *}$ and Dinggang Shen ${ }^{2,3,4}$ \\ ${ }^{1}$ School of Mathematics Science, Liaocheng University, Liaocheng, China, ${ }^{2}$ School of Biomedical Engineering, \\ ShanghaiTech University, Shanghai, China, ${ }^{3}$ Shanghai United Imaging Intelligence Co., Ltd., Shanghai, China, ${ }^{4}$ Department \\ of Artificial Intelligence, Korea University, Seoul, South Korea
}

Brain functional network (BFN) has become an increasingly important tool to explore individual differences and identify neurological/mental diseases. For estimating a "good" BFN (with more discriminative information for example), researchers have developed various methods, in which the most popular and simplest is Pearson's correlation (PC). Despite its empirical effectiveness, PC only encodes the low-order (second-order) statistics between brain regions. To model high-order statistics, researchers recently proposed to estimate BFN by conducting two sequential PCs (denoted as $P C^{2}$ in this paper), and found that $P C^{2}$-based BFN can provide additional information for group difference analysis. This inspires us to think about (1) what will happen if continuing the correlation operation to construct much higher-order BFN by $P C^{n}(n>2)$, and (2) whether the higher-order correlation will result in stronger discriminative ability. To answer these questions, we use $P C^{n}$-based BFNs to predict individual differences (Female vs. Male) as well as identify subjects with mild cognitive impairment $(\mathrm{MCl})$ from healthy controls (HCs). Through experiments, we have the following findings: (1) with the increase of $\mathrm{n}$, the discriminative ability of $P C^{n}$-based BFNs tends to decrease; (2) fusing the $P C^{n}$-based BFNs $(n>1)$ with the $P C^{1}$-based BFN can generally improve the sensitivity for $\mathrm{MCl}$ identification, but fail to help the classification accuracy. In addition, we empirically find that the sequence of BFN adjacency matrices estimated by $P C^{n}(n=1,2,3, \cdots)$ will converge to a binary matrix with elements of \pm 1 .

Keywords: brain functional network, Pearson's correlation, mild cognitive impairment, gender prediction, higher-order correlation

\section{INTRODUCTION}

Brain functional network (BFN), learning from the resting-state functional magnetic resonance imaging (rs-fMRI), has become an increasingly important tool to understand the brain working mechanism (Liu et al., 2015; Jiang et al., 2020; Xue et al., 2020; Chen et al., 2021), reveal the biomarkers of neurological/mental disorders (Bijsterbosch et al., 2017; Li et al., 2017; Liu et al., 2017; Sun et al., 2020), and predict the individual differences (Dubois and Adolphs, 2016). However, establishing a "good" BFN (with more discriminative information for example) is currently a challenging problem, due to the low-quality of fMRI data and the high complexity of our brain.

In the past decades, numerous BFN construction methods (McLntosh and Gonzalez-Lima, 1994; Marrelec et al., 2006; Ramsey et al., 2010; Qiao et al., 2016; Li et al., 2017; Jiang et al., 2019) have been proposed. The main difference among these methods lies in the calculation of the relationship 
between different brain regions of interest (ROIs). Pearson's correlation (PC) is the commonly used method for constructing BFN due to its relative simplicity and reliability (Smith et al., 2013).

However, traditional PC cannot capture the complex relationship (e.g., non-linearity and non-Gaussianity) among ROIs because it can only encode the simple linear correlation (Wan et al., 2006; Xie et al., 2008; Zhang et al., 2016). In contrast, the two sequential PCs (denoted as $P C^{2}$ in this paper) provide a high-order statistic that, in theory, can encode more complex relationship (Swami et al., 1997; Zhou et al., 2018). In fact, $P C^{2}$ has been used to estimate BFN and some findings have been obtained in recent years (Chen et al., 2016; Zhang et al., 2016; Zhou et al., 2018). For instance, Chen et al. (2016) revealed that $P C^{2}$ can encode the complex interactions between different ROIs, and Zhang et al. (2016) found that $P C^{2}$ is more sensitive to $\mathrm{AD}$ identification.

In order to have an intuitive explanation of the original loworder PC and high-order PC (i.e., $P C^{2}$ ), we provide a simple analogy via a friendship network in Figure 1, where each node corresponds to a person and each edge means existence of friendship between two nodes. As shown in Figure 1, we note that there is no direct edge between Node 1 and Node 2, meaning that these two persons have no relationship in a low-order form. However, from a high-order view, Nodes 1 and 2 may have some kinds of relationship since they share three common friends. Such a friend-sharing relationship (with similar friend circles) can be easily captured by the high-order PC, according to its definition (i.e., two sequential correlations). This indicates that the high-order PC can find more complex relationship that cannot be directly modeled in a low-order way.

Inspired by the potential powerfulness of $P C^{2}$, a natural question is (1) what will happen if continuing the correlation operation to construct much higher-order BFN by $P C^{n}(n>2)$, and (2) whether the higher-order correlation will result in stronger discriminative ability or other useful information.

For investigating the above problems, we construct $P C^{n}$-based BFNs on two public datasets, and then apply the constructed BFNs to conduct the classification task. Through the experiments, we get two interesting findings: (1) for the $P C^{n}$-based BFNs, with the increase of $n$, their discriminative ability tends to decrease; (2) by fusing the $P C^{n}$-based BFNs $(n>1)$ with the original $P C$-based $\mathrm{BFN}$, the sensitivity for MCI identification is generally increased, but the classification accuracy has no significant improvement. In addition, we have empirically found that the BFN adjacency matrix sequence constructed by $P C^{n}$ ( $n$ $=1,2,3 \ldots)$ is convergent, where $P C^{1}$ corresponds to the original PC.

The rest of this paper is organized as follows. In section 2, we introduce the data acquisition, data preprocessing and the $P C^{n}$-based BFN construction scheme. In section 3, we design two classification tasks for evaluating the proposed methods. In section 4, we discuss our findings and analyze the convergence of the $P C^{n}$-matrix sequence, and also introduce several limitations of this work with possible research plans in the future. Finally, we briefly summarize this paper in section 5 .

\section{MATERIALS AND METHODS}

\subsection{Data Acquisition}

Two public datasets, from Alzheimer's Disease Neuroimaging Initiative $(\mathrm{ADNI})^{1}$ and Human Connectome Project $(\mathrm{HCP})^{2}$, respectively, are used in our experiments. For ADNI dataset, we first remove the subjects if (1) the maximum translation/rotation of the head motion exceeds 2.0, or (2) the volumes with big frame-to-frame displacement $(>0.5)$ accumulate more than 2.5 min (Power et al., 2012), which leaves us 299 scans (including $154 \mathrm{HCs}$ and $145 \mathrm{MCIs}$ ) from 143 participants (with some participants scanned at one or more times). The scanning parameters are given as follows: the image resolution is from 2.29 to $3.31 \mathrm{~mm}$ for in-plane and the slice thickness equal to 3.31 $\mathrm{mm}$, TE (echo time) $=30 \mathrm{~ms}$, TR (repetition time) is from 2.2 to $3.1 \mathrm{~s}$, flip angle $=80^{\circ}$, field of view $(\mathrm{FOV})=198.75 \times 212$ $\mathrm{mm}^{2}$, matrix size $=64 \times 64$, voxel size $=3.3 \times 3.3 \times 3.3 \mathrm{~mm}^{3}$ and the scanning time for each subject is $7 \mathrm{~min}$ (resulting in 140 volumes) (Wang et al., 2019). The demographic information of 143 subjects is shown in Table 1.

For HCP dataset, 1,003 subjects, including 469 males and 534 females, are involved in our experiment. Each subject was scanned in four sessions, each of which generates 1,200 volumes, thus resulting in 4,800 volumes. Data acquisition is performed as follows: $\mathrm{TR}=720 \mathrm{~ms}$, $\mathrm{TE}=33.1 \mathrm{~ms}$ with 72 slices, flip angle $=52^{0}, \mathrm{FOV}=208 \times 180 \mathrm{~mm}^{2}$, matrix size $=104 \times 90$, and voxel size $=2 \times 2 \times 2 \mathrm{~mm}^{3}$ (WU-Minn, 2017). The demographic information of 1,003 subjects is shown in Table 2.

\subsection{Data Preprocessing}

For each subject from ADNI dataset, we discard the first three volumes for magnetization balance, and then use the FSL FEAT software ${ }^{3}$ for preprocessing the remaining 137 volumes, including slice timing correction, head motion estimation, bandpass filtering, and regression of nuisance covariates (white matter, cerebrospinal fluid, and motion parameters). After that, we align the fMRIs (that are skull-stripped based on T1-weighted MRI) onto the Montreal Institute of Neurology (MNI) space, and perform spatial smoothing by the Gaussian kernel with fullwidth-at-half-maximum (FWHM) of $6 \mathrm{~mm}$. Finally, we divide the preprocessed volumes into 116 ROIs based on the automatic anatomical labeling (AAL) template (Tzourio-Mazoyer et al., 2002), and extract its mean blood oxygen level dependent (BOLD) signals (fMRI series) (Wang et al., 2019).

For the subjects from HCP dataset, they are pre-processed according to WU-Minn (2017). More specifically, structural noise in the fMRI was removed through the pairsed independent component analysis (ICA) (Beckmann and Smith, 2004) with FMRIB's ICA-based Xnoiseifier (FIX) in FSL toolbox (Griffanti et al., 2014). Then, inter-subject registration of cerebral cortex was carried out using areal-feature-based alignment and Multimodal Surface Matching algorithm (MSMAII) (Smith et al., 2011; Glasser et al., 2016). In order to divide the ROIs of the brain,

\footnotetext{
${ }^{1}$ http://adni.loni.ucla.edu.

${ }^{2}$ http://www.humanconnectomeproject.org.

${ }^{3} \mathrm{http}: / /$ www.fmrib.ox.ac.uk/fsl.
} 


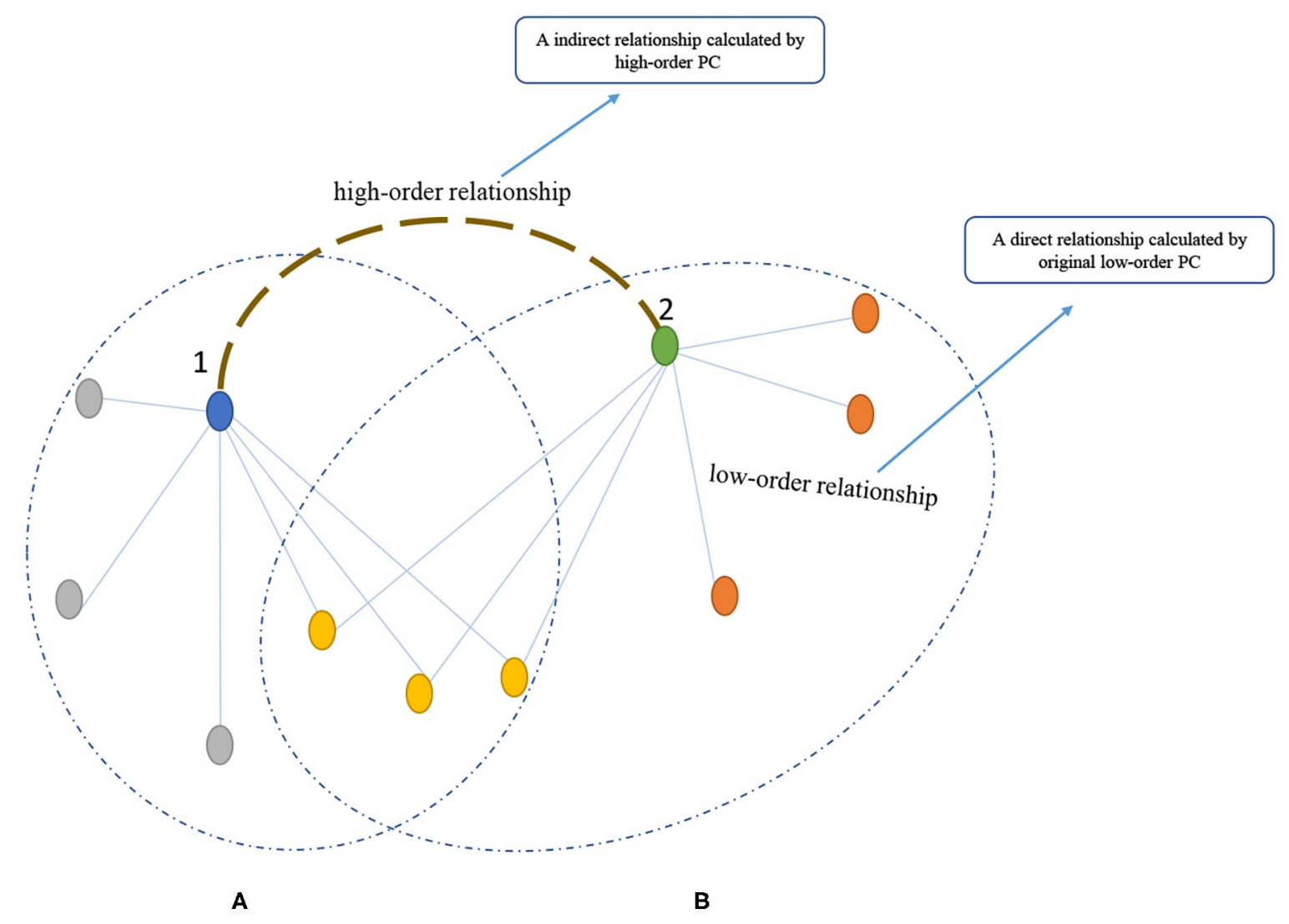

FIGURE 1 | An analogy of $P C^{2}$ to a friendship network. The $(\mathbf{A}, \mathbf{B})$ are the friendship networks of nodes 1 and 2 , respectively.

the data was first temporally normalized and fed into groupPCA (Beckmann and Smith, 2004; Smith et al., 2014), and then the group-ICA based on FSL's MELODIC tool (Griffanti et al., 2014) was applied to the data obtained by group-PCA (Beckmann and Smith, 2004). Note that the data dimension after group ICA determines the number of ROIs, which is simply set to 100 in this paper.

\subsection{PC-Based BFN Estimation}

As described earlier, $\mathrm{PC}$ is the simplest and most popular approach to construct BFNs (Smith et al., 2013). The edge weight between the ith and jth ROIs of $P C$-based BFN is defined as follows:

$$
r_{i j}=\frac{\left(x_{i}-\bar{x}_{i}\right)^{T}\left(x_{j}-\bar{x}_{j}\right)}{\sqrt{\left(x_{i}-\bar{x}_{i}\right)^{T}\left(x_{i}-\bar{x}_{i}\right)} \sqrt{\left(x_{j}-\bar{x}_{j}\right)^{T}\left(x_{j}-\bar{x}_{j}\right)}}
$$

where $x_{i} \in R^{M}$ is the fMRI signal from the ith ROI, and $\bar{x}_{i} \in R^{M}$ is a constant signal obtained by averaging the elements in $x_{i}$. Without loss of generality, we suppose that $x_{i}$ is centralized by $x_{i}-\bar{x}_{i}$ and normalized by $\sqrt{\left(x_{i}-\bar{x}_{i}\right)^{T}\left(x_{i}-\bar{x}_{i}\right)}$. Then, Equation (1) can be simplified to $r_{i j}=\left(x_{i}\right)^{T} x_{j}$, and its matrix form is defined as follows:

$$
R=X^{T} X
$$

TABLE 1 | The main demographic information of 143 subjects.

\begin{tabular}{lccc}
\hline Category & Scan \# & Age (Years) & Gender (M/F) \\
\hline $\mathrm{MCl}$ & 145 & $71.99 \pm 7.67$ & $95 / 50$ \\
$\mathrm{HC}$ & 154 & $75.36 \pm 6.16$ & $67 / 87$ \\
\hline
\end{tabular}

TABLE 2 | The main demographic information of 1, 003 subjects.

\begin{tabular}{lcc}
\hline Category & Scan \# & Age (Years) \\
\hline Female & 534 & $22-36+$ \\
Male & 469 & $22-36+$ \\
\hline
\end{tabular}

where $X=\left[x_{1}, x_{2}, \cdots, x_{N}\right] \in R^{(M \times N)}$ is the data matrix, and $R$ is the edge weight matrix of $P C$-based BFN. For the convenience of presentation in what follows, we simply call the original $\mathrm{PC}$ as $P C^{1}$, and the corresponding edge weight matrix of $P C$-based BFN as $R_{1}=R$.

\subsection{From $P C^{1}$ to $P C^{n}$}

Despite its simplicity and popularity, $P C^{1}$-BFN can only model the low-order statistics that may be insufficient to capture more complicated relationship between ROIs. As an alternative to $P C^{1}$, 


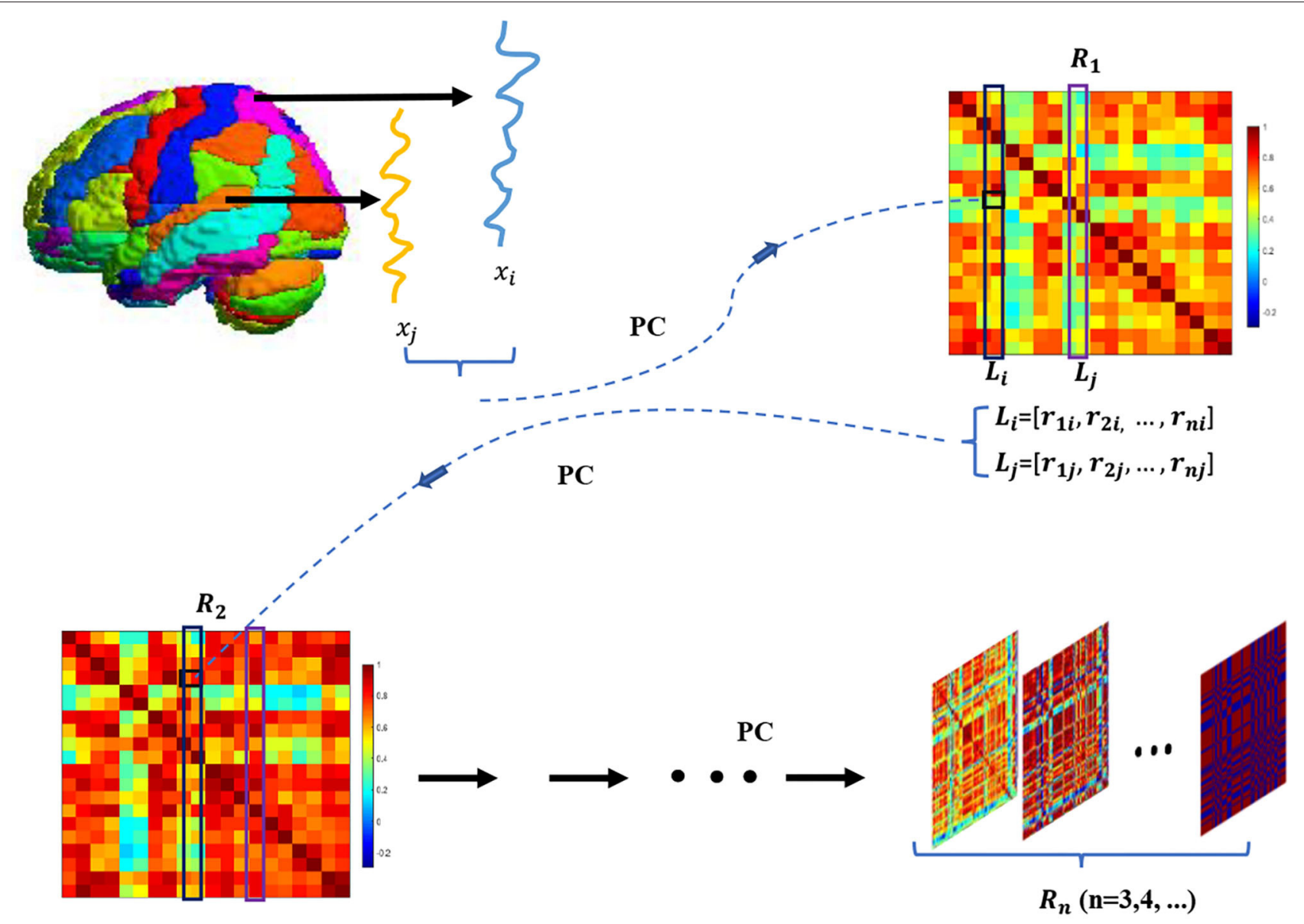

FIGURE 2 | The pipeline of building $R_{n} . R_{1}$ is first obtained via computing PC coefficient of the BOLD signals associated with different ROls. Then, $R_{1}$ is seen as the new data matrix to calculate $R_{2}$, where the feature vector $L_{i}$ is considered as a new "signal" that indicates the correlation between the ith ROI and other ROls. By continuously conducting the above operation, we calculate $R_{n}(n=2,3, \cdots)$.

Zhang et al. (2016) recently proposed to construct the highorder BFN by correlation's correlation. Specifically, as shown in Figure 2, the $P C^{1}$-based BFN (i.e., $R_{1}$ ) is first established by Equations ( 1 ) or ( 2 ). Then, the $R_{1}$ is used as a data matrix for constructing the high-order $P C^{2}$-based $B F N$ (i.e., $R_{2}$ ) by considering each row (or column) of $R_{1}$ as a new "signal" associated with each ROI. Interestingly, the researchers find that $R_{2}$ can provide additional information in group difference analysis (Zhang et al., 2016).

Motived by $P C^{2}$, in this paper we go further to construct higher-order $P C^{n}$-based BFN (i.e., $R_{n}, n>2$ ) by continuously conducting the correlation operation. As a result, we obtain a sequence of BFN adjacency matrices, and this sequence from $R_{1}$ to $R_{n}$ can be achieved by the following iteration formula (please see Appendix for the details of formula derivation):

$$
R_{n}=\frac{R_{n-1} C R_{n-1}}{\sqrt{\operatorname{diag}\left(R_{n-1} C R_{n-1}\right)} \sqrt{\operatorname{diag}\left(R_{n-1} C R_{n-1}\right)}}, n=2,3,4, \cdots
$$

where $C=I-\frac{E}{n}$ is a constant matrix (with $I$ and $E$ being the identity and all-one matrix, respectively), and $\operatorname{diag}(A)$ is a diagonal matrix that shares the same diagonal elements with $A$.

\section{EXPERIMENT}

In this section, we conduct experiments to answer the questions about the higher-order BFNs: (1) whether the higher-order BFN are more discriminative for achieving better classification performance, and (2) by combinating with $P C^{1}$-based $B F N$, whether the $P C^{n}$-BFNs $(n>2)$ can provide additional information as $P C^{2}$-based BFNs.

\subsection{Experimental Setting}

In this experiment, the classification performance is evaluated by five-fold subject-level cross validation (5-F CV), to ensure that fMRI scans of the subject do not appear in both training and testing sets (Wong and Tzu-Tsung, 2015). Specifically, as shown in Figure 3, the 143 (or 1,003) subjects of ADNI (or HCP) are first divided into five-fold, each of which contains almost the 

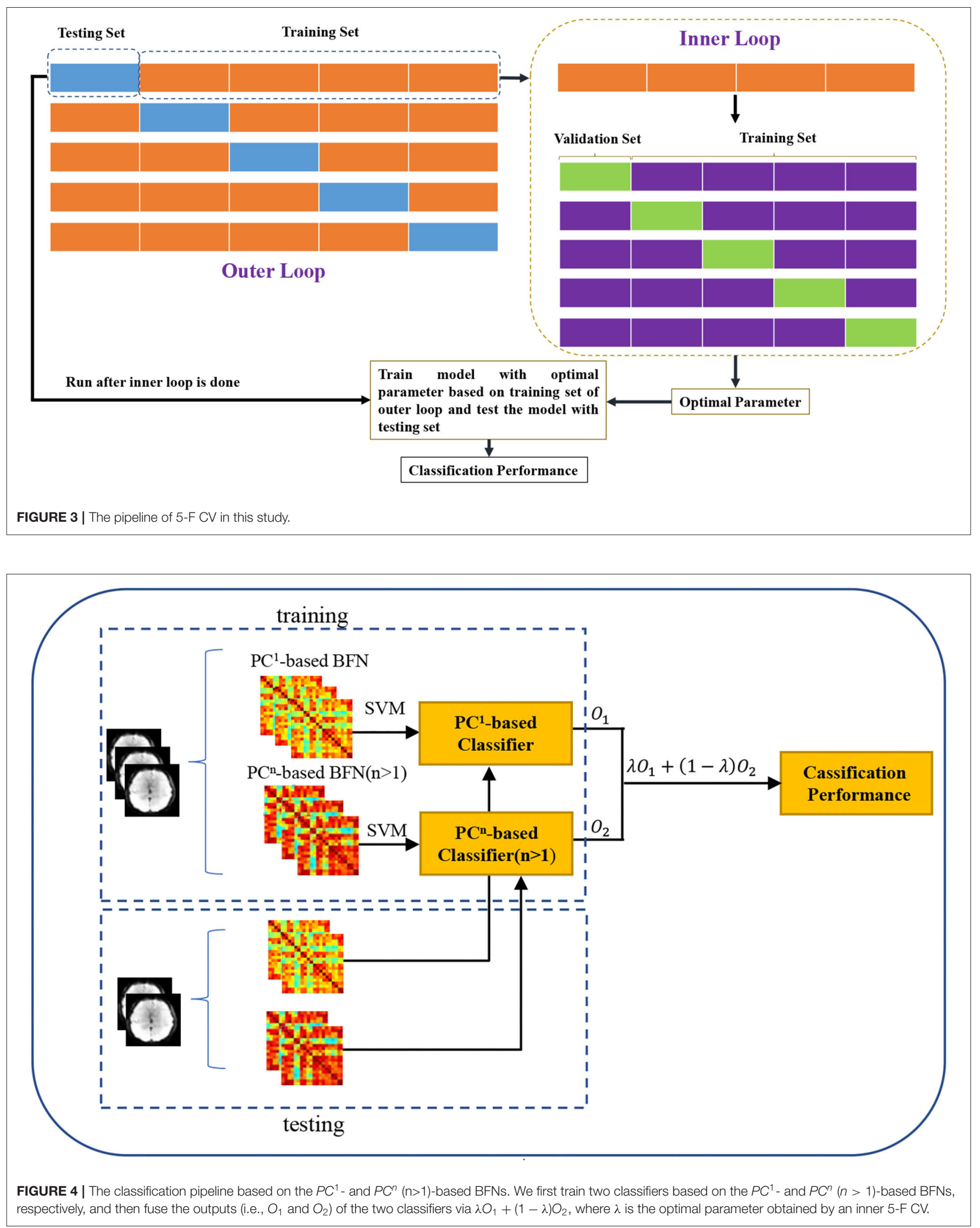


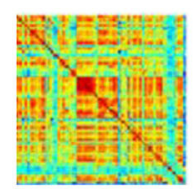

$\mathrm{PC}^{1}$

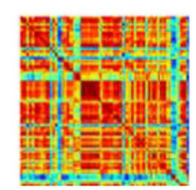

$\mathrm{PC}^{2}$

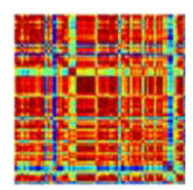

$\mathrm{PC}^{3}$

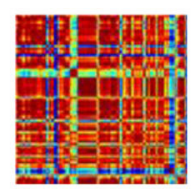

$\mathrm{PC}^{4}$

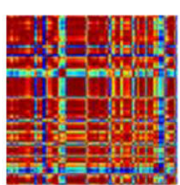

$\mathrm{PC}^{5}$

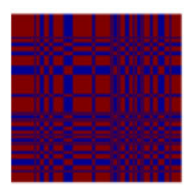

$\mathrm{PC}^{16}$

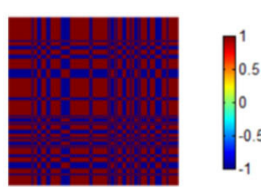

$\mathrm{PC}^{17}$

FIGURE 5 | The visualization of BFNs with different order $n$. We empirically note that the adjacency matrix sequence will converge after dozens of iterations.

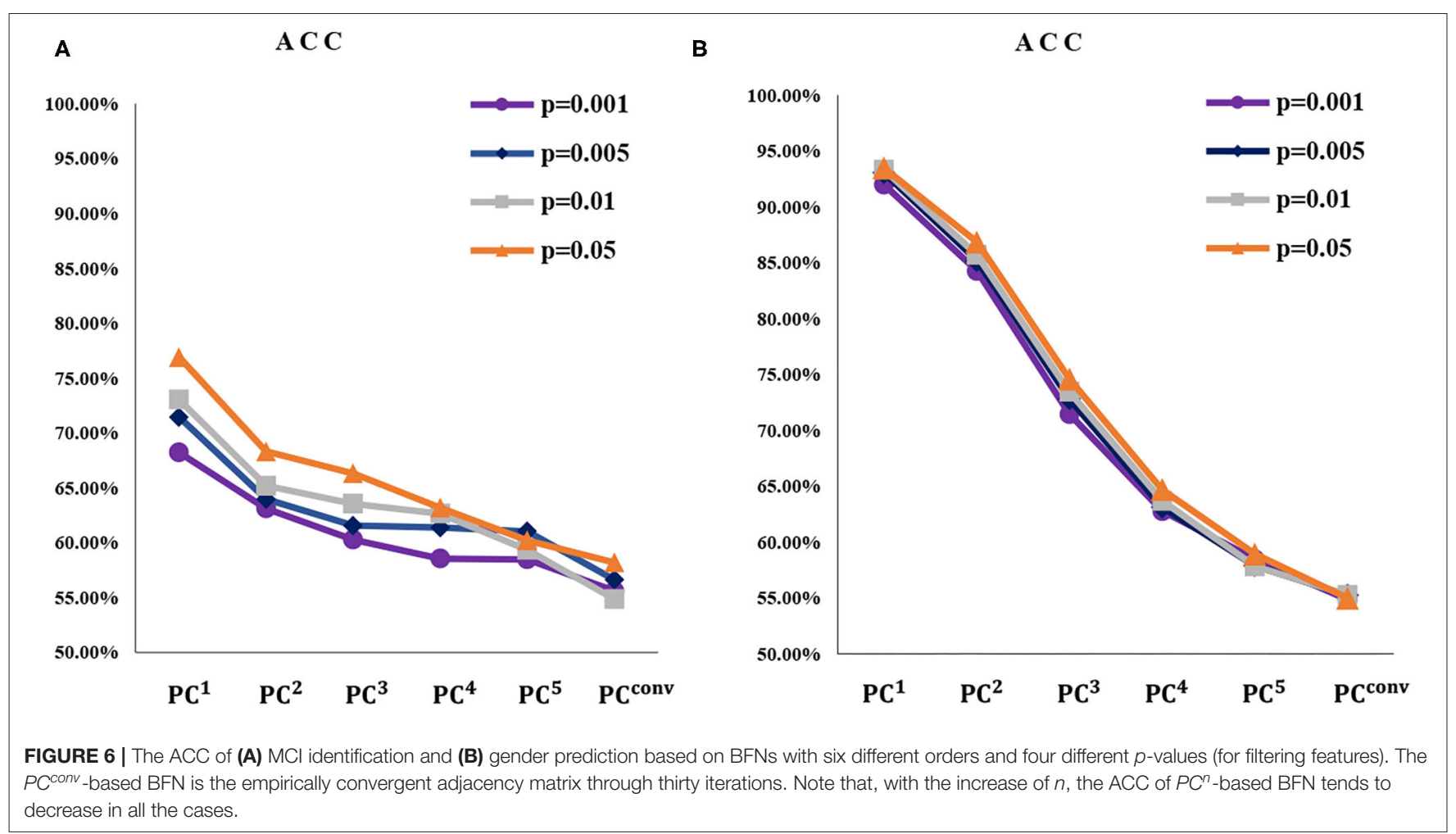

same number of subjects. Then, four-fold are used to train the classifier, and the remaining one is used to test the classification performance. Linear support vector machine (SVM) (Chang and Lin, 2011) with default parameter $C=1$ is utilized to perform the classification task. The edge weights of the BFN are adopted as features for classification. In consideration of the fact that the number of edges far exceeds the sample size, $t$-test is used to select edge weight features prior to the classification task. Two binary classification tasks are conducted to evaluate the constructed BFNs with different orders. One is to identify subjects with MCI from HCs based on the ADNI dataset, and the other is to predict gender based on the HCP dataset.

Furthermore, we design experiment to verify whether the $P C^{n}$-BFNs $(n>2)$ can provide additional information by combining the $P C^{n}$ - and $P C^{1}$-based BFNs. In particular, we first train two classifiers (SVM with $\mathrm{C}=1$ ) based on $P C^{1}$ - and $P C^{n}$ $(n>1)$-based BFNs, respectively, and then fuse the outputs of these two classifiers. As shown in Figure 4, the outputs, $O_{1}$ and $\mathrm{O}_{2}$, of the two classifiers measure the probabilities that the current subject belongs to the positive class. With the two probabilistic outputs, we can achieve the fused classification result via $\lambda O_{1}+(1-\lambda) O_{2}$, where the optimal parameter $\lambda$ is selected in the range of $[0.1,0.2,0.3,0.4, \cdots, 0.9]$ by an inner 5 -F CV on the training set. Finally, we retrain the classifier with the optimally selected parameter, and put the BFNs of the testing subject into the trained classifiers for the ultimate performance as shown in Figure 3.

\subsection{Estimated Functional Brain Networks}

In the first group of experiments, we visualize the sequence of BFN constructed by $P C^{n}$.

In Figure 5, we randomly select a subject from ADNI dataset and visualize the BFNs constructed by different methods. It can be observed that the BFNs estimated by $P C^{16}$ and $P C^{17}$ are almost the same, indicating that the adjacency matrix sequence may converge to a binary matrix with entries of 1 and -1 . This phenomenon motivates us to explore and prove it in theory. In the discussion section, we will further analyze and discuss this problem. 

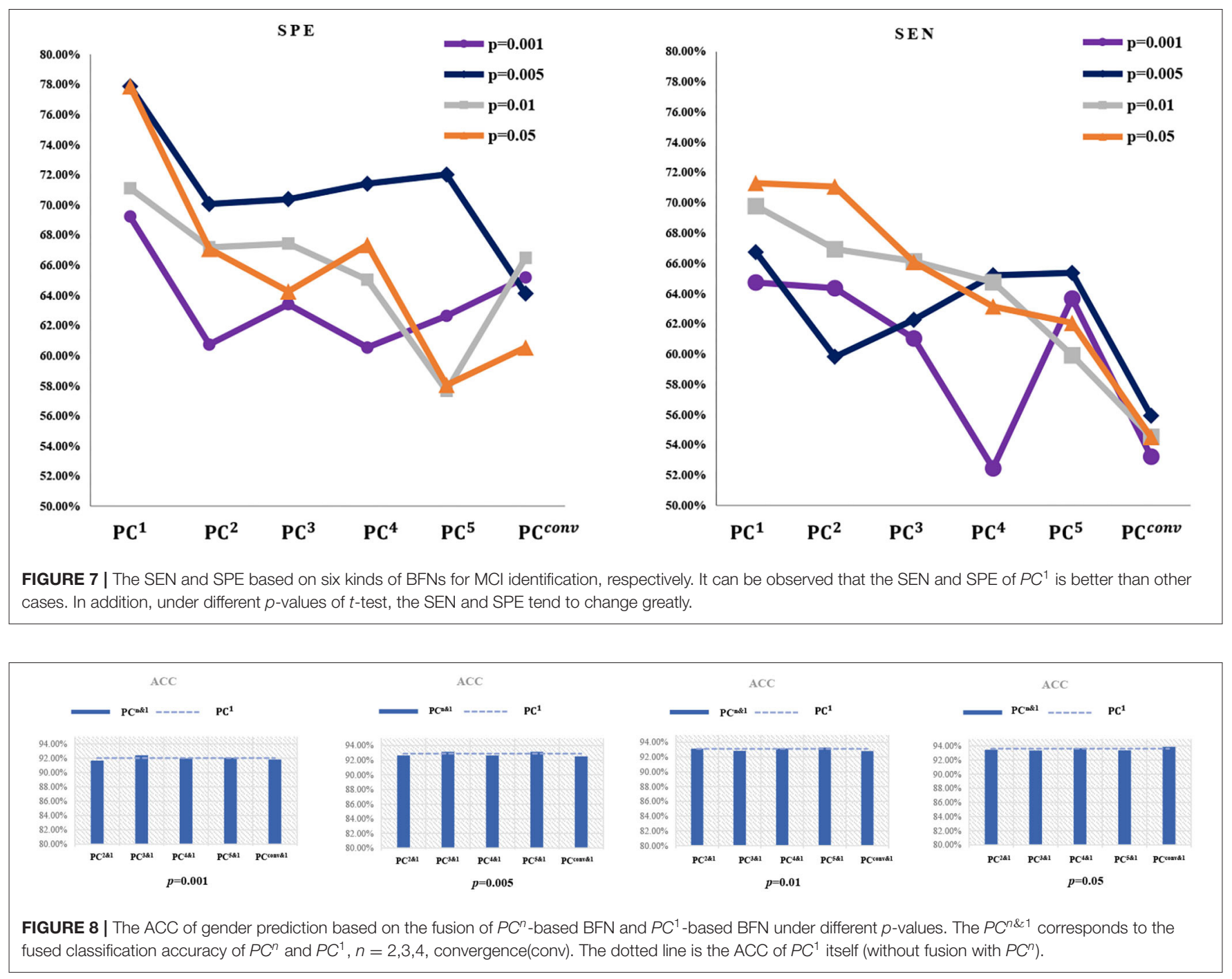

\subsection{Experiment Results}

In this group of experiments, two binary classification tasks (i.e., MCI vs. HC, Female vs. Male) based on the constructed BFNs with different orders are first conducted to evaluate whether the higher-order BFN are more discriminative, and then to verify whether the $P C^{n}$-BFNs $(\mathrm{n}>2)$ can provide additional information by combining the $P C^{n}$ - and $P C^{1}$-based BFNs. We evaluate the classification performance of different methods by a set of quantitative measures, including accuracy (ACC), sensitivity (SEN), and specificity (SPE). Their expressions are defined as follows:

$$
\begin{gathered}
A C C=\frac{T P+T N}{T P+T N+F N+F P} \\
S E N=\frac{T P}{T P+F N} \\
S P E=\frac{T N}{T N+F P}
\end{gathered}
$$

where TP, TN, FP, and FN denote the number of true positive, true negative, false positive, and false negative numbers, respectively.

As described in the experimental setting, we construct BFNs with different orders via multiple sequential PCs and apply them in the classification task. The classification results on two datasets are reported in Figures 6, 7.

Furthermore, the classification results based on the fusion of the low-order and higher-order BFNs are reported in Figures 8, 9, respectively.

\section{DISCUSSION}

In this section, we first study the discrimination of features and the additional information provided by higher-order BFN. Then, we further analyze the convergence of BFNs with different orders and show some related findings about it. Finally, we illustrate the limitations of the current work and the direction of future work. 


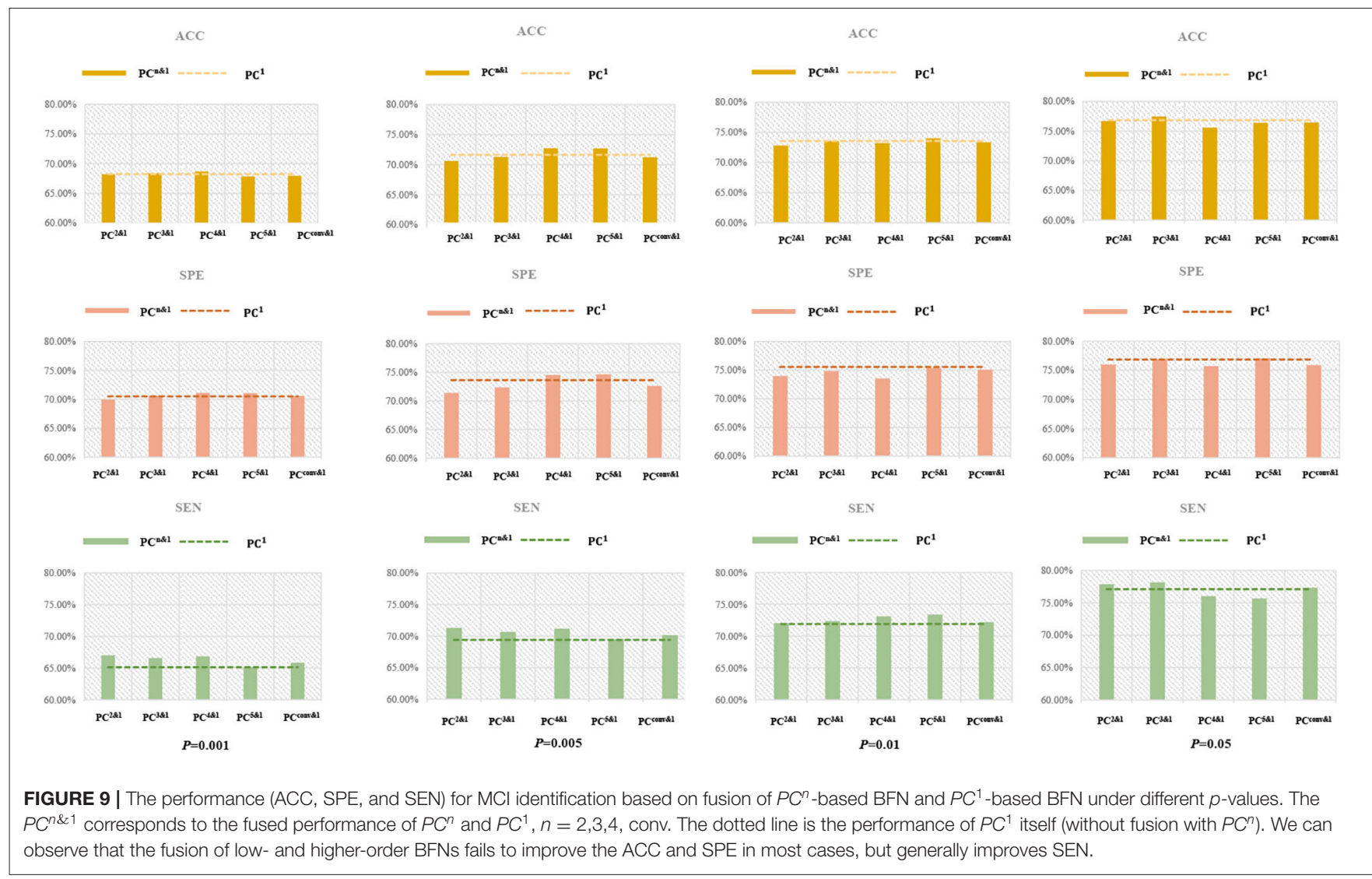

\subsection{Discrimination of Higher-Order BFN}

Based on the classification results, we analyze the discrimination of higher-order BFN. Based on Figures 6, 7.

(1) As $n$ increases, the ACC of $P C^{n}$ generally decreases for both MCI identification and gender prediction, even with various $p$-values in $t$-test for selecting different groups of feature. This illustrates that the higher-order does not result in stronger discriminative ability. Especially when the BFN converges with a big $\mathrm{n}$, the ACC approaches 50\%, meaning that its discrimination almost disappears since our task is a binary classification.

(2) Different from the ACC that decreases monotonically, the SPE and SEN generally fluctuate for the MCI identification task under most $p$-values. However, the overall performance shows a downtrend for both SPE and SEN. This further illustrates that the higher-order BFNs in the sense of multiple correlations do not necessarily help the discrimination.

(3) As a byproduct of our experiments, we find that the $p$ value generally has a great impact on the final performance. For example, as shown in Figure 6, the $P C^{n}$-based BFNs have better performance when the $p$-value is 0.05 . In terms of the fused classification results, it can also be observed that $p=0.05$ will result in the best ACC, SEN, and SPE, as shown in Figure 9. This suggests that we should carefully select features (edge weights) prior to the classification.

In order to better explain our classification results, we further investigate the number of features involved in the $P C^{n}$-based
MCI classification, and report the results with $p$-value of 0.001 in Figure 10.

It can be seen, from Figure 10, that $P C^{1}$ includes the largest number of features (207 features) and the number of features selected by $P C^{n}$ decrease with the increase of $n$. This means that the higher-order BFN instead contains less discriminative information than the low-order BFN.

\subsection{Supplementary Information of Higher-Order BFNs}

The higher-order BFNs can provide some additional information for the classification task. As shown in Figure 9, although it cannot benefit the ACC and SPE, the combination of $P C^{n}$-based BFN and $P C^{1}$-based BFN can generally improve the SEN. The improvement of SEN is generally important, because it may help to detect the brain disorders in their early stage. In addition, this also give a reason to believe that $P C^{n}(n>1)$ would provide potentially additional information for encoding complex relationship between different ROIs.

\subsection{Convergence Analysis}

In the process of constructing the BFN based on the sequential PCs, we have an interesting finding that the sequence of BFN adjacency matrices estimated by $P C^{n}(n=1,2,3 \cdots)$ will converge to a binary matrix with entries of 1 and -1 as shown in Figure 5. Although we cannot provide a direct explanation of this 


\section{0}

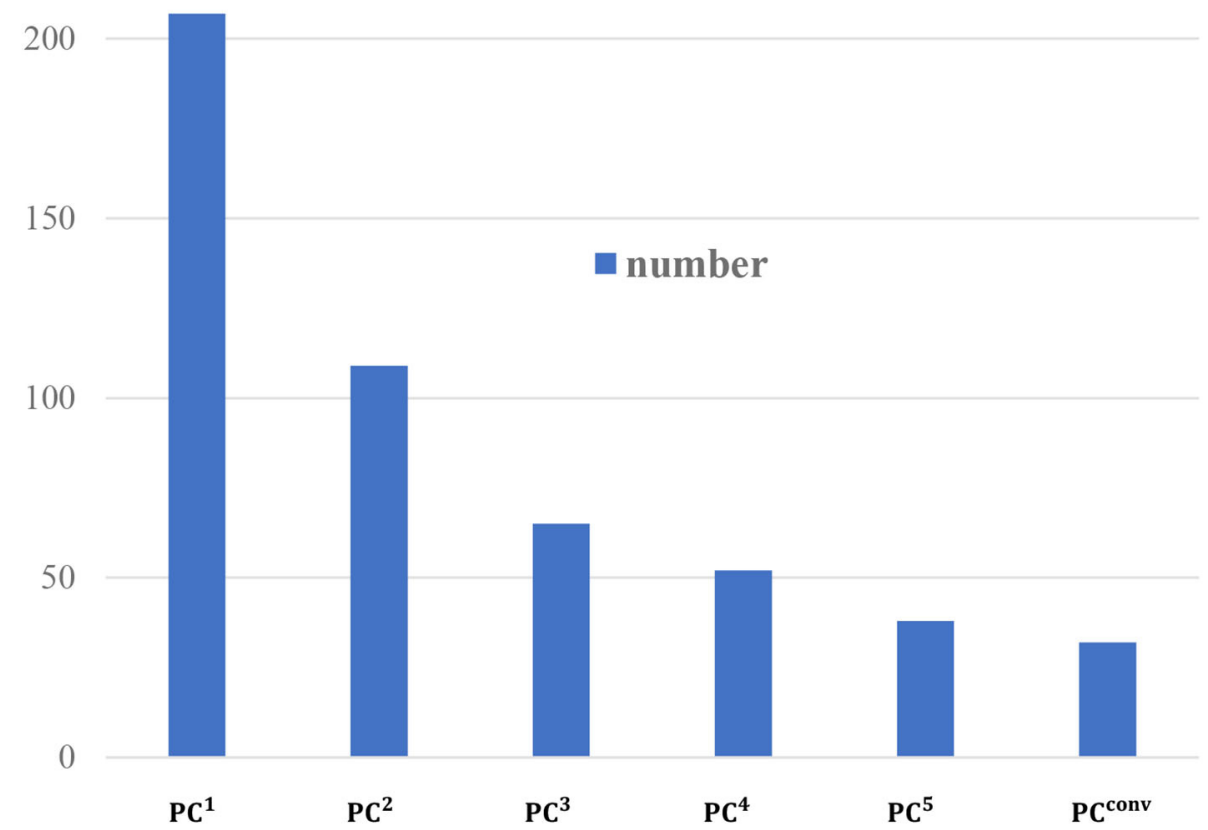

FIGURE 10 | The number of selected features with $p$-values $=0.001$.

phenomenon from a biological mechanism, it will inspire us to think whether the interaction between different ROIs promotes or inhibits each other at a certain working stage of the brain.

Currently, the convergence of the BFN sequence cannot be rigorously proved in theory. We will explore this problem in the future work. However, in the process of searching the rigorous proof of convergence, we achieve several interesting theoretical findings. In particular, let $\left\{R_{n}\right\}_{n=1}^{\infty}$ be the sequence of correlation coefficient matrices generated by the iterative formula shown in Equation ( 3 ), and then we have the following results or conjecture:

(1) After iterating the matrix for a certain number of times, the elements in the matrix will be monotonic with the number of iterations, and the sign of a small number of elements will change once.

(2) Supposing that the rank of $R_{n}$ is $r_{n}^{*}$, we can achieve a sequence of rank $\left\{r_{n}^{*}\right\}_{n=1}^{\infty}$ that is monotonically decreasing, since the rank of the matrix product is not greater than the rank of any one of them (Lancaster and Tismenetsky, 1985).

(3) According to Schauder's fixed-point theorem (Kellogg, 1976), we find that the iterative formula given in Equation ( 3 ) has a fixed-point ${ }^{4}$.

\footnotetext{
${ }^{4}$ Schauder fixed point: suppose $\mathrm{X}$ is a banach space, and $M \in X$ is a bounded closed convex set. If F: $M \rightarrow M$ is a compact-operator, there is $x \in M$ such that $\mathrm{F}(\mathrm{x})=\mathrm{x}$.
}

\subsection{Limitations and Future Work}

First, in this paper we only study the HOBFN constructed by multiple sequential PCs. In fact, however, many traditional BFN estimation methods such as sparse representation (Lee et al., 2011) can be extended to higher-order versions through the similar way (i.e., multiple sequential calculations). In the future, we plan to discuss more kinds of HOBFNs and systematically compare their discrimination. Second, we cannot rigorously prove the convergence of matrix sequence in theory, and the biological mechanism of HOBFN is unclear at present. We will try to prove its convergence and explore its biological mechanism in the future.

\section{CONCLUSION}

Recent researches have proposed many methods to construct high-order BFNs (HOBFNs) that can generally provide some additional information for poring cerebral mechanisms. For instance, Guo et al. (2017) constructed HOBFN using minimum spanning tree and Zhang et al. (2016) recently proposed to construct BFNs by conducting two sequential PCs. In this paper, we mainly focus on constructing the $P C^{n}$-based BFN by multiple sequential PCs, and exploring its performance in the classification tasks. Through experiments, we find that the higher-order does not necessarily result in stronger discriminative ability, but the additional information provided by $P C^{n}(\mathrm{n}>1)$ is helpful for $P C^{1}$ to 
improve the sensitivity of the classifier. In addition, we empirically find that the matrix sequence constructed by $P C^{n} \quad(n=1,2,3 \cdots)$ converges to a matrix with elements of -1 and 1 .

\section{DATA AVAILABILITY STATEMENT}

The original contributions generated for the study are included in the article/supplementary material, further inquiries can be directed to the corresponding author/s.

\section{ETHICS STATEMENT}

The studies involving human participants were reviewed and approved by public datasets HCP and ADNI. The

\section{REFERENCES}

Beckmann, C. F., and Smith, S. M. (2004). Probabilistic independent component analysis for functional magnetic resonance imaging. IEEE Trans. Med. Imag. 23, 137-152. doi: 10.1109/TMI.2003.822821

Bijsterbosch, J., Smith, S., and Beckmann, C. (2017). An Introduction to Resting State FMRI Functional Connectivity. Oxford University Press.

Chang, C., and Lin, C. (2011). Libsvm: a library for support vector machines. ACM Trans. Intell. Syst. Technol. 2, 1-27. doi: 10.1145/1961189.19 61199

Chen, H., Zhang, Y., Zhang, L., Qiao, L., and Shen, D. (2021). Estimating brain functional networks based on adaptively-weighted fMRI signals for MCI identification. Front. Aging Neurosci. 12:595322. doi: 10.3389/fnagi.2020.595322

Chen, X., Zhang, H., Gao, Y., Wee, C.-Y., Li, G., and Shen, D. (2016). High-order resting-state functional connectivity network for MCI classification. Hum. Brain Mapp. 37, 3282-3296. doi: 10.1002/hbm.23240

Dubois, J., and Adolphs, R. (2016). Building a science of individual differences from fMRI. Trends Cogn. Sci. 20, 425-443. doi: 10.1016/j.tics.2016.03.014

Glasser, M. F., Coalson, T. S., Robinson, E. C., Hacker, C. D., Harwell, J., Yacoub, E., et al. (2016). A multi-modal parcellation of human cerebral cortex. Nature 536, 171-178. doi: 10.1038/nature18933

Griffanti, L., Salimi-Khorshidi, G., Beckmann, C. F., Auerbach, E. J. Douaud, G., Sexton, C. E., et al. (2014). ICA-based artefact removal and accelerated fMRI acquisition for improved resting state network imaging. NeuroImage 95, 232-247. doi: 10.1016/j.neuroimage.2014.03.034

Guo, H., Lei, L., Chen, J., Xu, Y., and Xiang, J. (2017). Alzheimer classification using a minimum spanning tree of high-order functional network on fMRI dataset. Front. Neurosci. 11:639. doi: 10.3389/fnins.2017.00639

Jiang, W., Zhang, H., Zeng, L., Shen, H., and Shen, D. (2020). Dynamic neural circuit disruptions associated with antisocial behaviors. Hum. Brain Mapp. 42, 329-344. doi: 10.1002/hbm.25225

Jiang, X., Zhang, L., Qiao, L., and Shen, D. (2019). Estimating functional connectivity networks via low-rank tensor approximation with applications to MCI identification. IEEE Trans. Biomed. Eng. 67, 1912-1920. doi: 10.1109/TBME.2019.2950712

Kellogg, R. (1976). Uniqueness in the schauder fixed point theorem. Proc. Am. Math. Soc. 60, 207-210. doi: 10.1090/S0002-9939-1976-0423137-6

Lancaster, P., and Tismenetsky, M. (1985). The Theory of Matrices: With Applications. New York, NY: Academic Press.

Lee, H., Lee, D, S., Kang, H., Kim, B, N., and Chung, M. (2011). Sparse brain network recovery under compressed sensing. IEEE Trans. Med. Imag. 30, 1154-1165. doi: 10.1109/TMI.2011.2140380

Li, W., Wang, Z., Zhang, L., Qiao, L., and Shen, D. (2017). Remodeling Pearson's correlation for functional brain network estimation and autism spectrum disorder identification. Front. Neuroinformatics 11:55. doi: $10.3389 /$ fninf.2017.00055 patients/participants provided their written informed consent to participate in this study.

\section{AUTHOR CONTRIBUTIONS}

LQ and TG contributed to the conception and design of this research. LQ, YZ, and TG derived and designed the theoretical and experimental parts in the article. TG, LQ, YX, and DS wrote the first draft of the manuscript. All authors participated in the revision, reading, and approval of the manuscript.

\section{FUNDING}

This work was partly supported by National Natural Science Foundation of China (Nos. 61976110 and 11931008).
Liu, F., Guo, W., Fouche, J.-P., Wang, Y., Wang, W., Ding, J., et al. (2015). Multivariate classification of social anxiety disorder using whole brain functional connectivity. Brain Struct. Funct. 220, 101-115. doi: 10.1007/s00429-013-0641-4

Liu, F., Wang, Y., Li, M., Wang, W., Li, R., Zhang, Z., et al. (2017). Dynamic functional network connectivity in idiopathic generalized epilepsy with generalized tonic-clonic seizure. Hum. Brain Mapp. 38, 957-973. doi: $10.1002 / \mathrm{hbm} .23430$

Marrelec, G., Krainik, A., Duffau, H., Plgrini-Issac, M., Lehricy, S., Doyon, J., et al. (2006). Partial correlation for functional brain interactivity investigation in functional MRI. NeuroImage 32, 228-237. doi: 10.1016/j.neuroimage.2005.12.057

McLntosh, A. R., and Gonzalez-Lima, F. (1994). Structural equation modeling and its application to network analysis in functional brain imaging. Hum. Brain Mapp. 2, 2-22. doi: 10.1002/hbm.460020104

Power, J. D., Barnes, K. A., Snyder, A. Z., Schlaggar, B. L., and Petersen, S. E. (2012). Spurious but systematic correlations in functional connectivity MRI networks arise from subject motion. Neuroimage 59, 2142-2154. doi: 10.1016/j.neuroimage.2011.10.018

Qiao, L., Zhang, H., Kim, M., Teng, S., Zhang, L., and Shen, D. (2016). Estimating functional brain networks by incorporating a modularity prior. NeuroImage 141, 399-407. doi: 10.1016/j.neuroimage.2016.07.058

Ramsey, J. D., Hanson, S. J., Hanson, C., Halchenko, Y. O., Poldrack, R. A., and Glymour, C. (2010). Six problems for causal inference from FMRI. NeuroImage 49, 1545-1558. doi: 10.1016/j.neuroimage.2009.08.065

Smith, S. M., Hyvrinen, A., Varoquaux, G., Miller, K. L., and Beckmann, C. F. (2014). Group-PCA for very large fMRI datasets. NeuroImage 101, 738-749. doi: 10.1016/j.neuroimage.2014.07.051

Smith, S. M., Miller, K. L., Salimi-Khorshidi, G., Webster, M., Beckmann, C. F., Nichols, T. E., et al. (2011). Network modelling methods for FMRI. NeuroImage 54, 875-891. doi: 10.1016/j.neuroimage.2010.08.063

Smith, S. M., Vidaurre, D., Beckmann, C. F., Glasser, M. F., Jenkinson, M., Miller, K. L., et al. (2013). Functional connectomics from resting-state fMRI. Trends Cogn. Sci. 17, 666-682. doi: 10.1016/j.tics.2013.09.016

Sun, L., Xue, Y., Zhang, Y., Qiao, L., and Liu, M. (2020). Estimating sparse functional connectivity networks via hyperparameter-free learning model. Artif. Intell. Med. 111:102004. doi: 10.1016/j.artmed.2020.102004

Swami, A., Giannakis, G., B., Zhou, and G. (1997). Bibliography on higher-order statistics. Signal Process. 60, 65-126. doi: 10.1016/S0165-1684(97)00065-0

Tzourio-Mazoyer, N., Landeau, B., Papathanassiou, D., Crivello, F., Etard, O., Delcroix, N., et al. (2002). Automated anatomical labeling of activations in SPM using a macroscopic anatomical parcellation of the MNI MRI single-subject brain. NeuroImage 15, 273-289. doi: 10.1006/nimg.2001.0978

Wan, X., Riera, J., Iwata, K., Takahashi, M., Wakabayashi, T., and Kawashima, R. (2006). The neural basis of the hemodynamic response nonlinearity in human primary visual cortex: implications for neurovascular coupling mechanism. NeuroImage 32, 616-625. doi: 10.1016/j.neuroimage.2006.03.040 
Wang, M., Lian, C., Yao, D., Zhang, D., and Shen, D. (2019). Spatialtemporal dependency modeling and network hub detection for functional MRI analysis via convolutional-recurrent network. IEEE Trans. Bio-Med. Eng. 67, 2241-2252. doi: 10.1109/TBME.2019. 2957921

Wong, T.-T. (2015). Performance evaluation of classification algorithms by K-fold and leave-one-out cross validation. Pattern Recogn. 48, 2839-2846. doi: 10.1016/j.patcog.2015. 03.009

Wu-Minn, H. (2017). 1200 Subjects Data Release Reference Manual. Available online at: https://www.humanconnectome.org.

Xie, X., Cao, Z., and Weng, X. (2008). Spatiotemporal nonlinearity in resting-state fMRI of the human brain. NeuroImage 40, 1672-1685. doi: 10.1016/j.neuroimage.2008. 01.007

Xue, Y., Zhang, L., Qiao, L., and Shen, D. (2020). Estimating sparse functional brain networks with spatial constraints for MCI identification. PLoS ONE 15:e0235039. doi: 10.1371/journal.pone.02 35039

Zhang, H., Chen, X., Shi, F., Li, G., Kim, M., Giannakopoulos, P., et al. (2016). Topographical information-based high-order functional connectivity and its application in abnormality detection for mild cognitive impairment. J. Alzheimer's Dis. 54, 1095-1112. doi: 10.3233/JAD160092
Zhou, Y., Qiao, L., Li, W., Zhang, L., and Shen, D. (2018). Simultaneous estimation of low- and high-order functional connectivity for identifying mild cognitive impairment. Front. Neuroinform. 12:3. doi: 10.3389/fninf.2018.00003

Conflict of Interest: DS was employed by the company Shanghai United Imaging Intelligence Co., Ltd.

The remaining authors declare that the research was conducted in the absence of any commercial or financial relationships that could be construed as a potential conflict of interest.

Publisher's Note: All claims expressed in this article are solely those of the authors and do not necessarily represent those of their affiliated organizations, or those of the publisher, the editors and the reviewers. Any product that may be evaluated in this article, or claim that may be made by its manufacturer, is not guaranteed or endorsed by the publisher.

Copyright (c) 2021 Guo, Zhang, Xue, Qiao and Shen. This is an open-access article distributed under the terms of the Creative Commons Attribution License (CC BY).

The use, distribution or reproduction in other forums is permitted, provided the original author(s) and the copyright owner(s) are credited and that the original publication in this journal is cited, in accordance with accepted academic practice. No use, distribution or reproduction is permitted which does not comply with these terms. 


\section{APPENDIX}

\section{Construction Details of the Iterative \\ Formula}

Suppose that $R_{1}=\left(r_{i j}\right) \in R^{(n \times n)}$ is an initial Pearson's correlation coefficient matrix estimated from observed data of an $\mathrm{n}$-dimensional random vector $X=\left(X_{1}, X_{2}, \cdots, X_{n}\right)$. If considering $R_{1}$ as new observed data, each row (or equivalently each column, due to the symmetry) of which corresponds to a random variable in $X$, we can estimate another correlation coefficient matrix $R_{2}$ based on $R_{1}$. Further, by continuously conducting the operation of "correlation's correlation," we can have a sequence of correlation coefficient matrices $\left\{R_{n}\right\}_{n=1}^{\infty}$, and we empirically find that the matrix sequence will converge, if all the involved calculations are legal (for example, the denominator is not zero).

In order to prove the conjecture of convergence, we first express the sequence of correlation's correlation as an iterative formula. Specifically, let $r_{i}$ and $r_{j}$ be the $i t h$ and $j$ th rows of current correlation matrix $R=\left(r_{i j}\right) \in R^{(n \times n)}$, respectively. Then, the Pearson's correlation coefficient, denoted by $r_{i j}^{\text {new }}$, between the corresponding variables is given as follows,

$$
r_{i j}^{n e w}=\frac{\left(r_{i}-\overline{r_{i}} 1\right)\left(r_{j}-\overline{r_{j} 1}\right)^{T}}{\|\left(r_{i}-\overline{r_{i}} 1\|\| r_{j}-\overline{r_{j} 1} \|\right.}
$$

where $\bar{r}_{i}=\frac{\sum_{k=1}^{n} r_{i k}}{n}, \bar{r}_{j}=\frac{\sum_{k=1}^{n} r_{j k}}{n}(i, j=1,2, \cdots, n)$, and $1 \in$ $R^{(1 \times n)}$ is an all-one vector. As a result, the new correlation matrix $R^{\text {new }}=r_{i j}^{\text {new }}$ can be expressed as follows:

$$
\begin{aligned}
& R^{\text {new }}=\left[\begin{array}{c}
\frac{r_{1}-\bar{r}_{1} 1}{\left\|r_{1}-\bar{r}_{1} 1\right\|} \\
\vdots \\
\frac{r_{n}-\bar{r}_{n} 1}{\left\|r_{n}-\bar{r}_{n} 1\right\|}
\end{array}\right]\left[\left(\frac{r_{1}-\bar{r}_{1} 1}{\left\|r_{1}-\bar{r}_{1} 1\right\|}\right)^{T}, \cdots,\left(\frac{r_{n}-\bar{r}_{n} 1}{\left\|r_{n}-\bar{r}_{n} 1\right\|}\right)^{T}\right] \\
& =\left[\begin{array}{ccc}
\frac{1}{\left\|r_{1}-\bar{r}_{1} 1\right\|} & \cdots & 0 \\
\vdots & \ddots & \vdots \\
0 & \cdots & \frac{1}{\left\|r_{n}-\bar{r}_{n} 1\right\|}
\end{array}\right]\left[\begin{array}{c}
r_{1}-\bar{r}_{1} 1 \\
\vdots \\
r_{n}-\bar{r}_{n} 1
\end{array}\right] \\
& {\left[\left(r_{1}-\bar{r}_{1} 1\right)^{T}, \cdots,\left(r_{n}-\bar{r}_{n} 1\right)^{T}\right]\left[\begin{array}{ccc}
\frac{1}{\left\|r_{1}-\bar{r}_{1} 1\right\|} & \cdots & 0 \\
\vdots & \ddots & \vdots \\
0 & \cdots & \frac{1}{\left\|r_{n}-\bar{r}_{n} 1\right\|}
\end{array}\right]}
\end{aligned}
$$

Note that

$$
=\frac{1}{\left[\begin{array}{cccc}
\frac{1}{\left\|r_{1}-\bar{r}_{1} 1\right\|} & \cdots & 0 \\
\vdots & \ddots & \vdots \\
0 & \cdots & \frac{1}{\left\|r_{n}-\bar{r}_{n} 1\right\|}
\end{array}\right]}\left[\begin{array}{c}
\operatorname{diag}\left(\left[\begin{array}{c}
r_{1}-\bar{r}_{1} 1 \\
\vdots \\
r_{n}-\bar{r}_{n} 1
\end{array}\right]\left[\left(r_{1}-\bar{r}_{1} 1\right)^{T}, \cdots,\left(r_{n}-\bar{r}_{n} 1\right)^{T}\right]\right) \\
{\left[\begin{array}{c}
r_{1}-\bar{r}_{1} 1 \\
\vdots \\
r_{n}-\bar{r}_{n} 1
\end{array}\right]=R C=R\left(I-\frac{E}{n}\right)}
\end{array}\right.
$$

where $\operatorname{diag}(A)$ is a diagonal that shares the same diagonal elements with $A$, and $C=I-\frac{E}{n}$, with $I$ and $E$ being the identity and all-one matrix, respectively. Therefore, we have $R^{\text {new }}=\frac{1}{\sqrt{[\operatorname{diag}(R C R)]}}(R C R) \frac{1}{\sqrt{[\operatorname{diag}(R C R)]}}$, and accordingly have the iterative formula is given as follows below:

$$
R_{n}=\frac{1}{\sqrt{\left[\operatorname{diag}\left(R_{n-1} C R_{n-1}\right)\right]}}\left(R_{n-1} C R_{n-1}\right) \frac{1}{\sqrt{\left[\operatorname{diag}\left(R_{n-1} C R_{n-1}\right)\right]}}
$$

